\title{
Estimating relationships between phenotypes and subjects drawn from admixed families
}

Elizabeth M. Blue ${ }^{1 *}$, Lisa A. Brown², Matthew P. Conomos², Jennifer L. Kirk², Alejandro Q. Nato Jr ${ }^{1}$, Alice B. Popejoy ${ }^{4}$, Jesse Raffa ${ }^{3}$, John Ranola ${ }^{3}$, Ellen M. Wijsman ${ }^{1,2}$ and Timothy Thornton ${ }^{2}$

From Genetic Analysis Workshop 19

Vienna, Austria. 24-26 August 2014

\begin{abstract}
Background: Estimating relationships among subjects in a sample, within family structures or caused by population substructure, is complicated in admixed populations. Inaccurate allele frequencies can bias both kinship estimates and tests for association between subjects and a phenotype. We analyzed the simulated and real family data from Genetic Analysis Workshop 19, and were aware of the simulation model.

Results: We found that kinship estimation is more accurate when marker data include common variants whose frequencies are less variable across populations. Estimates of heritability and association vary with age for longitudinally measured traits. Accounting for local ancestry identified different true associations than those identified by a traditional approach. Principal components aid kinship estimation and tests for association, but their utility is influenced by the frequency of the markers used to generate them.

Conclusions: Admixed families can provide a powerful resource for detecting disease loci, as well as analytical challenges. Allele frequencies, although difficult to adequately estimate in admixed populations, have a strong impact on the estimation of kinship, ancestry, and association with phenotypes. Approaches that acknowledge population structure in admixed families outperform those which ignore it.
\end{abstract}

\section{Background}

Estimates of the kinship coefficient, defined as the probability that 2 alleles randomly sampled from 2 subjects are identical by descent, have many uses [1]. These include verifying pedigrees and sample identity [2], and tests for association [3]. Pruning markers for linkage disequilibrium (LD) [3] and minor allele frequency (MAF) improves kinship estimation. Sequence data offers additional challenges by discovering novel and very rare variants without accurate MAFs. We investigate kinship estimators and subsets of whole genome sequence (WGS) data from Genetic Analysis Workshop 19 (GAW19) to examine bias and accuracy.

\footnotetext{
*Correspondence: em27@uw.edu

'Division of Medical Genetics, Department of Medicine, University of Washington, Seattle, WA 98195, USA

Full list of author information is available at the end of the article
}

We explored whether genetic associations with systolic blood pressure (SBP) changed over time using longitudinal data. We estimated heritability and performed a kinship-adjusted half-genome-wide association test at the first and third visits for real SBP. We discuss the similarities, differences, and potential foundations for those changes.

Through both selection and drift, different populations can have different variants influencing the same trait, or very different frequencies of shared risk alleles. Admixture mapping takes advantage of these differences to identify risk loci [4]. Using the simulated SBP phenotype, we compare the power of RFMix, an accurate admixture mapping approach [4], to a traditional association test.

When testing for association between genotype and phenotype, principal components (PCs) are often included as covariates to minimize the effects of population 
structure. We investigated how well PCs estimated on different subsets of the data were able to capture ancestry proportions. Performance was evaluated with the coefficient of determination.

\section{Methods}

\section{Genetic map and markers}

We used GAW19 genome-wide association study (GWAS) data for odd-numbered autosomes for association testing and admixture mapping. Data were available on 959 subjects from 20 pedigrees. Sexaveraged map positions (cM) were converted by the Haldane map function from the Rutgers framework map with all of the Single Nucleotide Polymorphism database 134 (dbSNP134) variants [5]. The GAW19 WGS data for odd-numbered autosomes for 464 subjects from 20 pedigrees that passed the Support Vector Machines (SVM) filter and were missing $10 \%$ or less data were extracted with VCFtools [6]. Sexaveraged positions (cM) were linearly interpolated for the WGS data using the GWAS markers as a framework panel. We extracted exomes from WGS data using the 1000genomes Phase3-like BED file (http:// www.1000genomes.org/category/exome).

\section{Kinship estimation}

We pruned WGS variants for LD in PLINK [7] $\left(r^{2} \leq 0.2\right)$, applied filters described below, and pruned for LD with SNPRelate $[8]\left(r^{2} \leq 0.1\right)$. The Agnostic design includes every 100th variant: 21,484 WGS variants. Agnostic variants are rare: $58 \%$ have founder MAF of $1 \%$ or less. The Selective design includes variants with MAF $5 \%$ or greater: 64,389 WGS and 7215 exome variants. With an allele frequency spectrum comparable to the Selective design, our Homogenizing design includes the 30,710 WGS variants with alternate allele frequencies that minimally vary across the African (AFR), Native American (AMR), Asian (ASN), and European (EUR) populations [9] (maximum difference/ overall frequency $\leq 2$ ). This is similar to an approach to reduce bias caused by population structure [10].
Within SNPRelate, we applied three estimators: method of moments (MoM [7]), maximum likelihood (MLE [1, 3]) for non-inbred pairs, and robust Kinshipbased INference for Genome-wide association studies (KING [2]). No monomorphic single-nucleotide polymorphisms (SNPs) were evaluated and MAFs were estimated from the sample. The fourth estimator, PCRelate [11], is a moment estimator that adjusts for population structure using PCs, estimated here from the GWAS data using PC-AiR [11] with the pedigreebased kinship values. We report the weighted average of chromosome-specific estimates, with negative values set to zero. We evaluate how often each estimator would assign pairs of subjects to their pedigreebased relationship by rounding to the nearest expected value of kinship for unrelated pairs, first-, second-, third-, or fourth-degree relatives in an outbred pedigree (represented by gold bars in Fig. 1).

\section{Association testing and longitudinal analysis of systolic blood pressure data-real data}

We examined the role age may play on genetic effects on SBP by fitting a linear mixed-effects model to the longitudinal data for 916 individuals and 2189 SBP observations. Using the pedigree-based kinship matrix, we estimated the additive variance as a function of cubic splines of age, with $\log$ of the environmental variance similarly fit. SBP was adjusted using fixed effects for medication use, smoking status and gender, as well as smoking status and gender-specific curves for age.

We performed a half-genome scan for association with adjusted SBP at the first and third visits using Efficient Mixed-Model Association eXpedited (EMMAX) [12]. EMMAX is a mixed-model approach that calculates and uses an empirical genetic relatedness matrix (GRM) to account for both relatedness and population structure with a variance component for additive polygenic effects. A conservative Bonferroni correction for the association tests is $1.05 \times 10^{-7}(0.05 / 472,049$ markers $)$.

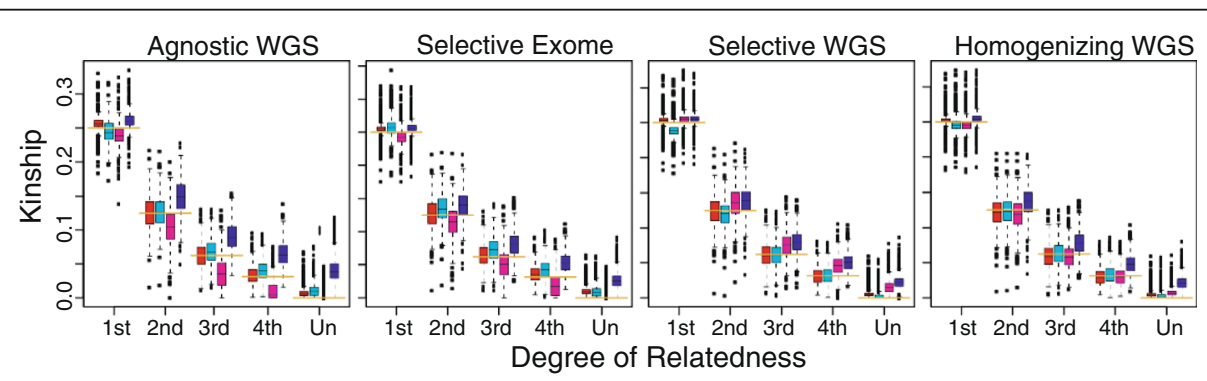

Fig. 1 Box-plot comparison of pairwise kinship estimates from WGS vs. exome data. Blue, PLINK method of moments; cyan, PC-Relate; magenta, KING-robust; red, maximum likelihood; Un, unrelated; WGS, whole genome sequence 


\section{Association testing-simulated data}

Admixture and association analysis of the simulated SBP at the first visit, adjusted as described above, included 955 subjects with GWAS data. Local ancestry was estimated by RFMix [4]. We phased the samples and imputed missing genotypes using Beagle3 [13] with 112 European (CEU [Northern Europeans from Utah]), 147 Yoruban (YRI [Yoruba in Ibadan, Nigeria]) and 63 Human Genome Diversity Project (HGDP) Native American (AMR [Admixed American]) samples [14, 15]. We included the 242,566 markers shared by GAW19 and reference panels. Proportion of ancestry from reference populations is an average of local ancestry values. Relatedness among subjects was included as a kinship matrix, $\phi$, estimated by PC-Relate so as to adjust for the proportion of total ancestry from each reference population. We fit a linear mixed model at each marker, assuming the trait is Normally distributed with a mean equal to an intercept plus a main effect for ancestry and variance $\phi \sigma_{\mathrm{g}}+I \sigma_{\mathrm{e}}$, where $I$ is the identity matrix. Ancestry for CEU, YRI, and AMR were each fit separately. For admixture mapping, we use twice the whole-genome-wide nominal $p$ value of $7 \times 10^{-6}$, which has previously shown a type I error of 0.05 [16]. For association mapping, we fit the same model using allelic SNP dosage as the predictor and apply the same Bonferroni threshold as for the analysis of real data, $1.05 \times 10^{-7}$.

\section{Population structure}

We began with the WGS data for 102 unrelated subjects from the GAW19 pedigrees. We created four subsets of variants based upon their MAF: rare (MAF $<0.01$ or $<0.05$ ) and common (MAF $>0.01$ or $>0.05$ ). There were $7,407,452$ SNPs (MAF $<0.05=5,803,244$ SNPs; MAF $<0.01=$ $4,522,880 \mathrm{SNPs})$. We estimated $\mathrm{R}^{2}$, the coefficient of determination, from a linear regression model with $10 \mathrm{PCs}$ from a PC analysis (PCA) as predictors and CEU, AMR, and YRI ancestry proportions from a supervised ADMIXTURE [17] analysis as the response. Details on the supervised ADMIXTURE analysis are described elsewhere [18]. We performed a PCA with a GRM, $\Psi$, with $(i, j)^{\text {th }}$ entry

$$
\psi_{i j}=\frac{\sum_{s=1}^{S}\left(\left(G_{i}^{s}-2 \hat{p}_{s}\right)\left(G_{j}^{s}-2 \hat{p}_{s}\right)\right)}{\sum_{s=1}^{S} 2 \hat{p}_{s}\left(1-\hat{p}_{s}\right)}
$$

where $S$ is the number of variants, $G_{i}^{s}$ and $G_{i}^{s}$ are the number of minor alleles $(0,1$, or 2$)$ that individuals $i$ and $j$ have at marker $s$, and $p_{\mathrm{s}}$ is the MAF at marker $s$. Unlike the EIGENSTRAT method [19], the entries in this GRM are calculated using ratios of sums, so low-frequency variants do not distort results.

\section{Results}

\section{Kinship estimation}

The MoM approach and Agnostic design provide the least-reliable kinship estimates (see Fig. 1), whereas the MLE values were consistently accurate. WGS and exome data performed comparably within the Selective design, whereas the Homogenizing design appeared to be more precise. MLE, KING, and PC-Relate performed similarly under the Homogenizing design. Excluding the Agnostic approach, the MLE and PC-Relate were each able to correctly assign relationships for more than $90 \%$ of pairs of first- and second-degree relatives and unrelated subjects (Table 1). Distant relationships cannot be reliably determined by any method.

\section{Association testing and longitudinal analysis of systolic blood pressure data-real data}

Environmental variance generally increased as a function of age, shown in the narrow-sense heritability estimates presented in Fig. 2. In those subjects who also had genotype data ( $N=831 ; 2060$ SBP measurements), we found the heritability estimates were robust to kinship estimators (KING, PC-Relate, and pedigree-based). The adjusted first- and third-visit SBP values are quite different, with a correlation of 0.48 , and the heritability estimate from EMMAX for the first-visit SBP is 0.26 and 0.13 for the third-visit SBP.

Table 1 Rate of successfully classified relationships. Frequency pairs within each relationship are correctly assigned to this degree of relationship

\begin{tabular}{|c|c|c|c|c|c|c|}
\hline \multirow[b]{2}{*}{ Design } & \multirow[b]{2}{*}{ Estimator } & \multicolumn{5}{|c|}{ Degree of relationship } \\
\hline & & $1 \mathrm{st}$ & 2nd & $3 r d$ & 4th & Unrelated \\
\hline \multirow[t]{4}{*}{ Agnostic WGS } & MLE & $99.7 \%$ & $91.0 \%$ & $76.1 \%$ & $60.2 \%$ & $99.7 \%$ \\
\hline & MoM & $100 \%$ & $93.4 \%$ & $54.4 \%$ & $16.9 \%$ & $14.5 \%$ \\
\hline & KING & $98.9 \%$ & $65.2 \%$ & $30.5 \%$ & $14.9 \%$ & $100 \%$ \\
\hline & $\begin{array}{l}\text { PC- } \\
\text { Relate }\end{array}$ & $99.7 \%$ & $94.1 \%$ & $81.9 \%$ & $57.9 \%$ & $93.9 \%$ \\
\hline \multirow[t]{4}{*}{ Selective WGS } & MLE & $99.7 \%$ & $92.7 \%$ & $76.3 \%$ & $61.3 \%$ & $99.9 \%$ \\
\hline & MoM & $100 \%$ & $97.4 \%$ & $78.4 \%$ & $38.1 \%$ & $58.0 \%$ \\
\hline & KING & $100 \%$ & $96.7 \%$ & $80.9 \%$ & $47.3 \%$ & $86.3 \%$ \\
\hline & $\begin{array}{l}\text { PC- } \\
\text { Relate }\end{array}$ & $99.5 \%$ & $91.8 \%$ & $78.4 \%$ & $62.6 \%$ & $100 \%$ \\
\hline \multirow[t]{4}{*}{ Selective exome } & MLE & $99.7 \%$ & $93.2 \%$ & $80.2 \%$ & $62.6 \%$ & $99.2 \%$ \\
\hline & MoM & $99.7 \%$ & $96.5 \%$ & $71.6 \%$ & $33.0 \%$ & $43.4 \%$ \\
\hline & $\mathrm{KING}$ & $99.5 \%$ & $80.0 \%$ & $52.4 \%$ & $30.5 \%$ & $99.5 \%$ \\
\hline & $\begin{array}{l}\text { PC- } \\
\text { Relate }\end{array}$ & $99.7 \%$ & $96.5 \%$ & $79.8 \%$ & $52.4 \%$ & $97.1 \%$ \\
\hline \multirow{4}{*}{$\begin{array}{l}\text { Homogenizing } \\
\text { WGS }\end{array}$} & MLE & $99.5 \%$ & $91.0 \%$ & $74.2 \%$ & $57.3 \%$ & $100 \%$ \\
\hline & MoM & $99.5 \%$ & $96.3 \%$ & $79.6 \%$ & $44.9 \%$ & $61.7 \%$ \\
\hline & KING & $99.5 \%$ & $88.1 \%$ & $73.3 \%$ & $59.5 \%$ & $99.9 \%$ \\
\hline & $\begin{array}{l}\text { PC- } \\
\text { Relate }\end{array}$ & $99.7 \%$ & $92.7 \%$ & $79.6 \%$ & $62.0 \%$ & $100 \%$ \\
\hline
\end{tabular}




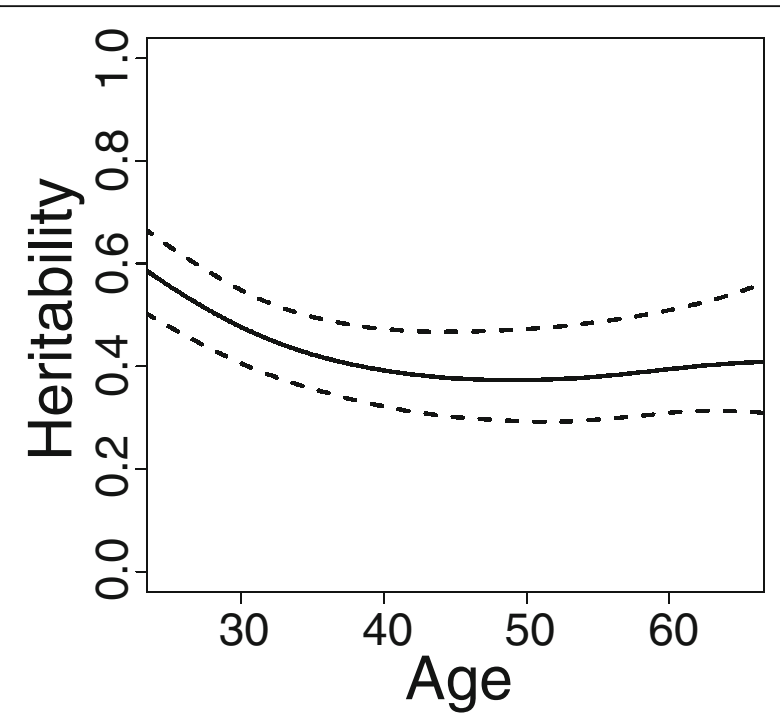

Fig. 2 Heritability estimate from longitudinal analysis as a function of age. Mean baseline age was 39.0 years $(S D=16.3)$ with a median follow-up of 10.4 years (Q1-Q3: 1.8-14.4). Number of SBP observations in each age (in years) strata: $<30: 483,[30,40): 415,[40,50): 438,[50,60)$ : 326, $\geq 60: 324$. The upper/lower dotted lines represent $95 \%$ confidence bands

The EMMAX approach found no significant associations with SBP at the first visit (Fig. 3a), but identified several for the third visit (Fig. 3b). Although there was some inflation of the test statistics for the third visit (genomic control inflation factor, $\lambda=1.03$ ), this was not seen for the first visit $(\lambda=1.002)$. The string of extreme $p$ values in Fig. 3b could be caused by an outlier. We repeated EMMAX analyses without the subject with the most extreme SBP at the third visit (Fig. 3c). Although $\lambda$ did not change much $(\lambda=1.034)$, the association signals on chromosomes $1,9,11$, and 13 were eliminated. The remaining signal on chromosome (chr) three remained, where the top SNP (rs7637973, in the LRRC31 gene) had a $p$ value of $5 \times 10^{-10}$.

\section{Association testing-simulated data}

Admixture mapping had no power at the significance level of $1.4 \times 10^{-5}$. If we reduce the significance threshold to $5 \times 10^{-4}$, we have $17 \%$ power across 200 simulated replicates to detect the variant in the P2RX5 gene on chr17 and $81 \%$ power to detect the variant in the COL5A3 gene on chr19. For association mapping, we observed $100 \%$ power across 200 replicates to detect the causal variant in the MAP4 gene on chr3 at the significance level of $10^{-7}$. Figure 4 shows the distribution of $p$ values for each approach for the first simulated replicate.

\section{Population structure}

As shown in Table 2, the MAF of marker data influenced our ability to capture population substructure. PCA with rare variants captured the YRI structure substantially better than using common variants, whereas common variants captured CEU/AMR ancestry better than the rare variants. The boundary between common and rare variation ( $1 \%$ vs. $5 \%$ MAF) had relatively minor influence on results.

\section{Discussion}

We have shown that the frequency of alleles included in kinship, association, and population structure estimation have strong influences on their results. Kinship estimation is most accurate when markers are restricted to common variants that are not ancestry informative, and the moment estimator showing the least bias incorporated ancestry-informative PCs. Admixture mapping and association testing each identified different causal genes for the simulated adjusted SBP, likely a consequence of differences in frequency of risk alleles in the AMR and CEU reference populations. This warrants a future analysis evaluating a single test of ancestry at each locus using a 2-degreesof-freedom test, as opposed to treating each ancestry separately. When estimating population structure, the marker data must be selected using the frequency of

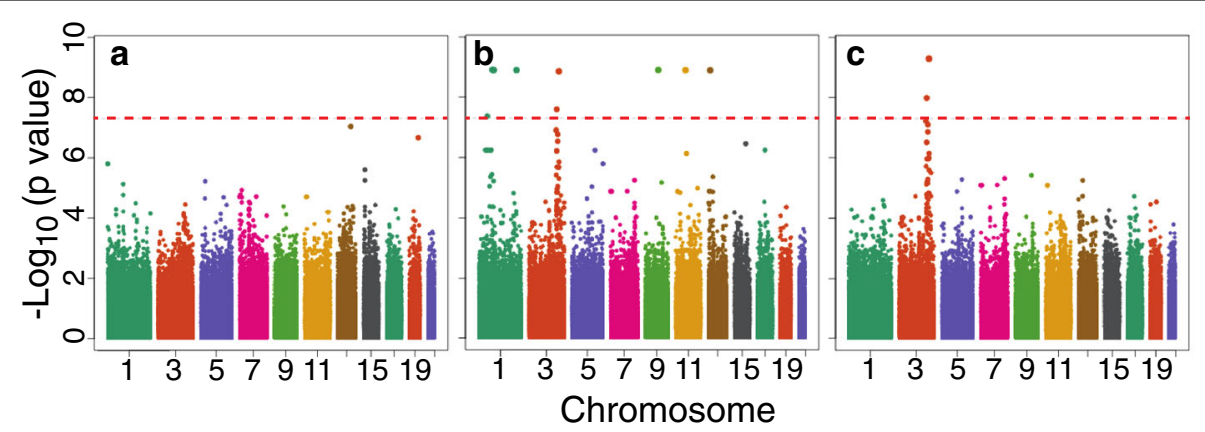

Fig. 3 EMMAX association test results for real first (a) and third (b) visit adjusted SBP, and third visit excluding an outlier (c). Bonferroni-corrected significance threshold is shown with a horizontal line 


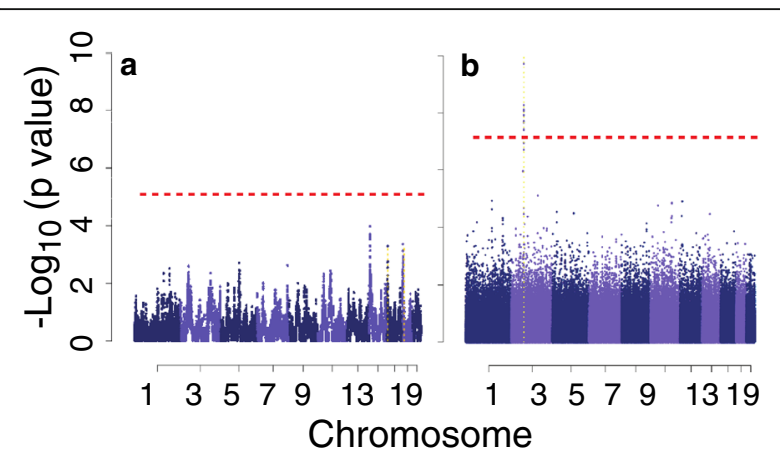

Fig. 4 Admixture mapping (a) and association testing (b) for adjusted simulated SBP using local ancestry. Significance thresholds are shown with a red horizontal line, based on theory [16] (a) or Bonferroni correction (b)

alleles in multiple relevant reference populations in order to adequately capture the complexity of ancestry in admixed populations.

Association testing found little evidence for real adjusted SBP loci, and the inflation of EMMAX results with third-visit SBP was likely caused by the increase in environmental variance in the trait as age increased, consistent with the change in heritability estimates over time. There could be shared environmental factors acting on third-visit SBP that are not being modeled. This would cause the systematic inflation of test statistics across the genome.

\section{Conclusions}

Variants with imprecise allele frequencies bias estimates of kinship, PCA, and association testing. Admixture

Table 2 Principal components analysis with rare and common WGS variants

\begin{tabular}{|c|c|c|c|c|c|c|c|c|}
\hline & \multirow[b]{2}{*}{ PCs } & \multirow[b]{2}{*}{ MAF } & \multicolumn{3}{|c|}{ PCA-Seq } & \multicolumn{3}{|c|}{ EIGENSTRAT } \\
\hline & & & CEU & AMR & YRI & CEU & AMR & YRI \\
\hline \multirow[t]{4}{*}{$\mathrm{R}^{2}$ for rare variants } & \multirow[t]{2}{*}{10} & $<0.01$ & 0.18 & 0.16 & 0.33 & 0.17 & 0.15 & 0.33 \\
\hline & & $<0.05$ & 0.79 & 0.80 & 0.55 & 0.78 & 0.79 & 0.54 \\
\hline & \multirow[t]{2}{*}{20} & $<0.01$ & 0.42 & 0.42 & 0.43 & 0.42 & 0.42 & 0.42 \\
\hline & & $<0.05$ & 0.83 & 0.84 & 0.66 & 0.82 & 0.83 & 0.65 \\
\hline \multirow{4}{*}{$\begin{array}{l}\mathrm{R}^{2} \text { for common } \\
\text { variants }\end{array}$} & \multirow[t]{2}{*}{10} & $<0.01$ & 0.95 & 0.95 & 0.13 & 0.95 & 0.95 & 0.17 \\
\hline & & $<0.05$ & 0.95 & 0.95 & 0.13 & 0.95 & 0.95 & 0.11 \\
\hline & \multirow[t]{2}{*}{20} & $<0.01$ & 0.97 & 0.97 & 0.22 & 0.97 & 0.97 & 0.41 \\
\hline & & $<0.05$ & 0.97 & 0.97 & 0.22 & 0.97 & 0.97 & 0.23 \\
\hline \multirow[t]{2}{*}{$R^{2}$ for all variants } & 10 & - & 0.95 & 0.96 & 0.18 & 0.96 & 0.96 & 0.22 \\
\hline & 20 & - & 0.97 & 0.97 & 0.41 & 0.97 & 0.97 & 0.49 \\
\hline
\end{tabular}

MAF minor allele frequency, $\mathrm{R}^{2}$, the coefficient of determination from a linear regression model with 10 PCs included as predictors and the proportion of CEU, AMR, and YRI ancestry proportions from ADMIXTURE analysis as the response mapping and association testing proved complementary. The influence of environment on estimates of heritability and association appear to have been revealed by analysis of longitudinal data.

\section{Declarations}

This research was supported by the National Institutes of Health $(\mathrm{NIH})$, grants AG040184, AG005136, AG049507, MH094293, and GM046255. The Genetic Analysis Workshops are supported by NIH grant GM031575.

This article has been published as part of BMC Proceedings Volume 10 Supplement 7, 2016: Genetic Analysis Workshop 19: Sequence, Blood Pressure and Expression Data. Summary articles. The full contents of the supplement are available online at http://bmcproc.biomedcentral.com/ articles/supplements/volume-10-supplement-7. Publication of the proceedings of Genetic Analysis Workshop 19 was supported by National Institutes of Health grant R01 GM031575.

\section{Authors' contributions}

All authors participated in study design and analysis. EMB drafted the manuscript, and all authors edited and approved the final manuscript.

\section{Competing interests}

The authors declare they have no competing interests.

\section{Author details}

'Division of Medical Genetics, Department of Medicine, University of Washington, Seattle, WA 98195, USA. ${ }^{2}$ Department of Biostatistics, University of Washington, Seattle, WA 98195, USA. ${ }^{3}$ Department of Statistics, University of Washington, Seattle, WA 98195, USA. ${ }^{4}$ Institute for Public Health Genetics, University of Washington, Seattle, WA 98195, USA.

Published: 18 October 2016

\section{References}

1. Milligan BG. Maximum-likelihood estimation of relatedness. Genetics. 2003; 163(3):1153-67.

2. Manichaikul A, Mychaleckyj JC, Rich SS, Daly K, Sale M, Chen WM. Robust relationship inference in genome-wide association studies. Bioinformatics. 2010;26(22):2867-73

3. Choi Y, Wijsman EM, Weir BS. Case-control association testing in the presence of unknown relationships. Genet Epidemiol. 2009;33(8):668-78

4. Maples BK, Gravel S, Kenny EE, Bustamante CD. RFMix: a discriminative modeling approach for rapid and robust local-ancestry inference. Am J Hum Genet. 2013;93(2):278-88.

5. Matise TC, Chen F, Chen W, De La Vega FM, Hansen M, He C, Hyland FC, Kennedy GC, Kong X, Murray SS, et al. A second-generation combined linkage physical map of the human genome. Genome Res. 2007;17(12):1783-6.

6. Danecek P, Auton A, Abecasis G, Albers CA, Banks E, DePristo MA, Handsaker RE, Lunter G, Marth GT, Sherry ST, et al. The variant call format and VCFtools. Bioinformatics. 2011;27(15):2156-8.

7. Purcell $S$, Neale B, Todd-Brown $K$, Thomas L, Ferreira MA, Bender D, Maller J, Sklar P, de Bakker PI, Daly MJ, et al. PLINK: a tool set for whole-genome association and population-based linkage analyses. Am J Hum Genet. 2007;81(3):559-75.

8. Zheng X, Levine D, Shen J, Gogarten SM, Laurie C, Weir BS. A highperformance computing toolset for relatedness and principal component analysis of SNP data. Bioinformatics. 2012;28(24):3326-8.

9. 1000 Genomes Project Consortium, Abecasis GR, Altshuler D, Auton A, Brooks LD, Durbin RM, Gibbs RA, Hurles ME, McVean GA. A map of human genome variation from population-scale sequencing. Nature. 2010; 467(7319):1061-73.

10. Morrison J. Characterization and correction of error in genome-wide IBD estimation for samples with population structure. Genet Epidemiol. 2013; 37(6):635-41.

11. Conomos MP, Miller MB, Thronton TA. Robust inference of population structure for ancestry prediction and correction of stratification in the presence of relatedness. Genet Epidemiol. 2015;39(4):276-93.

12. Kang HM, Sul JH, Service SK, Zaitlen NA, Kong SY, Freimer NB, Sabatti C, Eskin E. Variance component model to account for sample structure in genome-wide association studies. Nat Genet. 2010;42(4):348-54. 
13. Browning BL, Browning SR. A unified approach to genotype imputation and haplotype-phase inference for large data sets of trios and unrelated individuals. Am J Hum Genet. 2009;84(2):210-23.

14. Cann HM, de Toma C, Cazes L, Legrand MF, Morel V, Piouffre L, Bodmer J, Bodmer WF, Bonne-Tamir B, Cambon-Thomsen A, et al. A human genome diversity cell line panel. Science. 2002;296(5566):261-2.

15. International HapMap Consortium. The International HapMap Project. Nature. 2003:426(6968):789-96.

16. Tang $\mathrm{H}$, Coram M, Wang P, Zhu X, Risch N. Reconstructing genetic ancestry blocks in admixed individuals. Am J Hum Genet. 2006;79(1):1-12.

17. Alexander DH, Novembre J, Lange K. Fast model-based estimation of ancestry in unrelated individuals. Genome Res. 2009;19(9):1655-64.

18. Thornton TA, Conomos MP, Sverdlov S, Blue EM, Cheung CY, Glazner CG, Lewis SM, Wijsman EM. Estimating and adjusting for ancestry admixture in statistical methods for relatedness inference, heritability estimation, and association testing. BMC Proc. 2014;8 Suppl 1:S5.

19. Price AL, Patterson NJ, Plenge RM, Weinblatt ME, Shadick NA, Reich D. Principal components analysis corrects for stratification in genome-wide association studies. Nat Genet. 2006;38(8):904-9.

\section{Submit your next manuscript to BioMed Central} and we will help you at every step:

- We accept pre-submission inquiries

- Our selector tool helps you to find the most relevant journal

- We provide round the clock customer support

- Convenient online submission

- Thorough peer review

- Inclusion in PubMed and all major indexing services

- Maximum visibility for your research

Submit your manuscript at www.biomedcentral.com/submit 